\title{
Eye and appearance characteristics of encephalocraniocutaneous lipomatosis
}

\author{
Yanming $\operatorname{Tian}^{1} \cdot$ Yi Wang $^{2} \cdot$ Xiaowei Gao ${ }^{1} \cdot$ Yulong Zhang ${ }^{1} \cdot$ Yan Ju$^{1}$
}

Received: 8 August 2018 / Accepted: 15 August 2018 / Published online: 24 September 2018

(c) The Royal College of Ophthalmologists 2018

Encephalocraniocutaneous lipomatosis (ECCL) is a rare neurocutaneous syndrome. The unique clinical manifestations of the disease include ocular edema or hamartoma, abnormal skin on the head and neck, and corresponding hair loss on the scalp. Historical literature on ECCL consists mainly of case reports and does not present the common features of the disease. We present the characteristic manifestations of the eye and skin to provide ophthalmologists with a fuller understanding of in ECCL.

\section{Clinical information}

Four patients, all male, aged 3-18 years, presented with ophthalmologic disorders caused by local or diffuse hyperplasia of the ocular surface and had irregular granule-like lesions on the head or neck and unilateral visible granular raised lesions on the tongue mucosal surface (Fig. 1). No brain abnormality was apparent on skull computed tomography, and focal calcification was visible on the wall of the eye. All patients had normal intelligence and no history of

The study was approved by the institutional review board of $\mathrm{xxx} 474 \mathrm{th}$ hospital, the patient's inform consent was obtained.

Yanming Tian

tianyanming@163.com

$\triangle$ Xiaowei Gao

Gaoxiaowei@163.com

$\triangle$ Yulong Zhang

zhangyl112@163.com

$\triangle$ Yan Ju

juyan474@163.com

1 No. 474 Hospital of the Chinese People's Liberation Army (PLA), Urumqi 830012, China

2 Orbital Disease Institute, Armed Police General Hospital, Beijing 100039, China seizures. ocular surface tumor was removed with conjunctival sac reconstruction or lamellar keratoplasty.

Pathological results: Two cases were corneal Sebaceous tumor (mature squamous tumor) and two cases were fibrous epithelial polyps.

\section{Discussion}

ECCL is a rare neurocutaneous syndrome with unknown etiology and multiple sporadic differences in ethnicity, gender, and family history. Since Haberland [1] described this syndrome in 1970, more than 60 instances of related disorders have been published worldwide, mainly reported via pediatrics, and neurosurgery cases [2, 3]. The main features of the syndrome include asymmetric skin damage to the head and neck, central nervous system abnormalities(CNS), ocular abnormalities, often bilaterally. hair loss in the local skin lesion area. The most common eye lesions in ECCL are ocular tumors around the eyeball and small skin nodules. Other rare eye abnormalities of the syndrome such as bilateral optic disk defects and lack of bilateral iris eyelid retraction have been reported [4].

The occurrence of ECCL may be related to genetic variation, and it is speculated that autosomal gene mutations lead to ectodermal dysplasia, while chimerism may be the cause of mesenchymal tumors and angiogenesis.

Syndromes that need to be differentiated from linear nevus sebaceous syndrome (LNS), and oculocerebrocutaneous syndrome (OCC). A diagnosis of OCC needs to include CNS cysts or hydrocephalus, small eyeballs with orbital cysts. Skin lesions of LNS are mainly located in the midline of the face forming a linear pattern. According to the revised ECCL diagnostic criteria (Table 1) by Moog [5] in 2009, The ECCL diagnosis of these four cases is clear.

This group of patients shared the following common clinical features of ECCL: (1) ocular lesions; (2) flaky granular pigmentation of the area of skin loss; (3) granular mucosa visible on the surface of the tongue not 
Fig. 1 Appearance of ECCL. A: Case 1. Left eye

subconjunctival multifocal pink raised lesions. B: Case 2. Right corneal surface and left subconjunctival pink focal, smooth, bulging lesion. C: Case 3. Pink corneal smooth bulging lesion on the left cornea.

D: Case 4. right eye conjunctiva, and substantial freshly appearing protrusion on the right side of

the skin. E,F: case 1 and case 2 . Side view: left scalp, face, and neck skin lesions and hairless area. G: case 4. Black lesions on the ipsilateral neck. H: Granular bulge of the left tongue mucosa
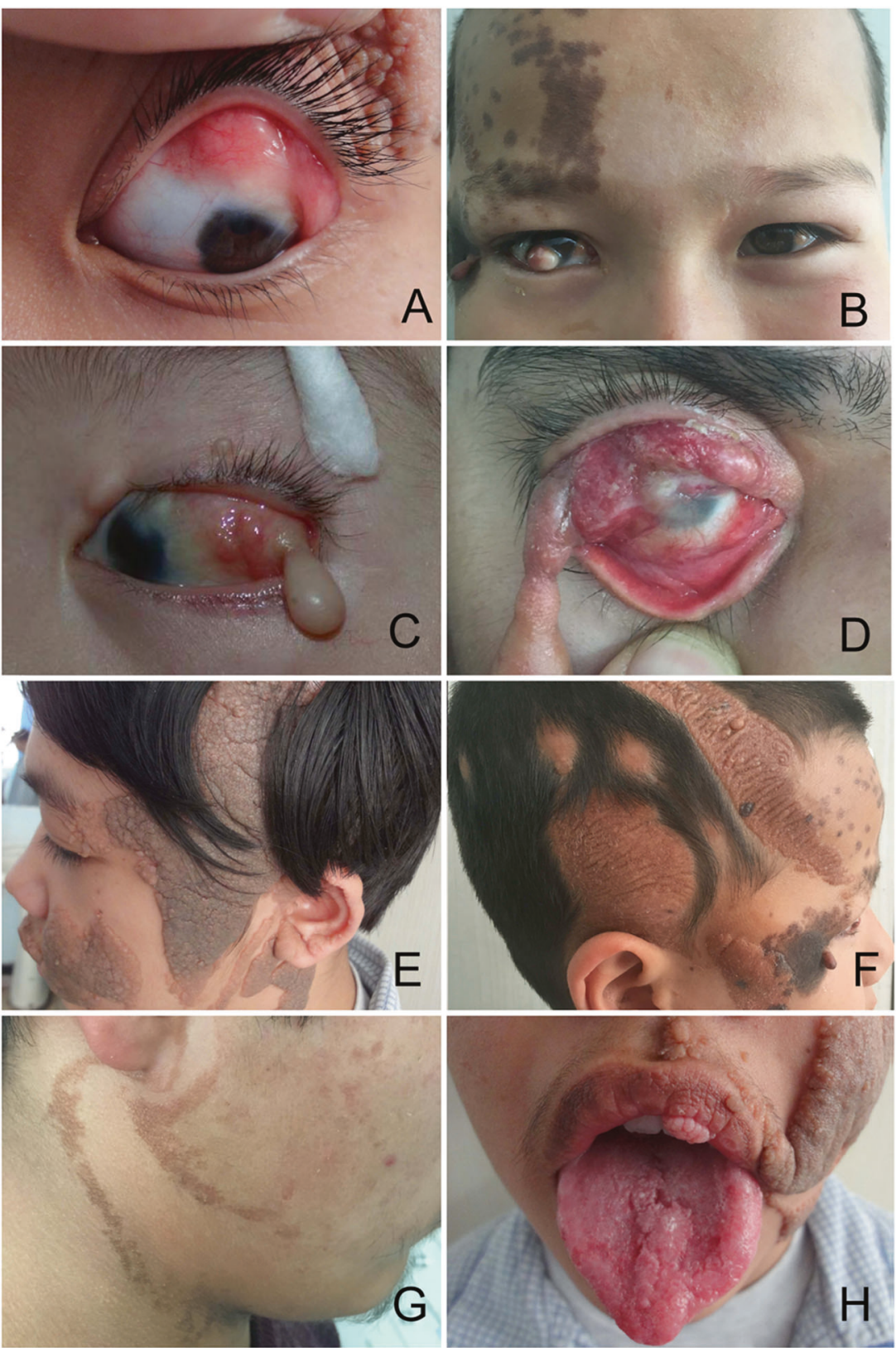


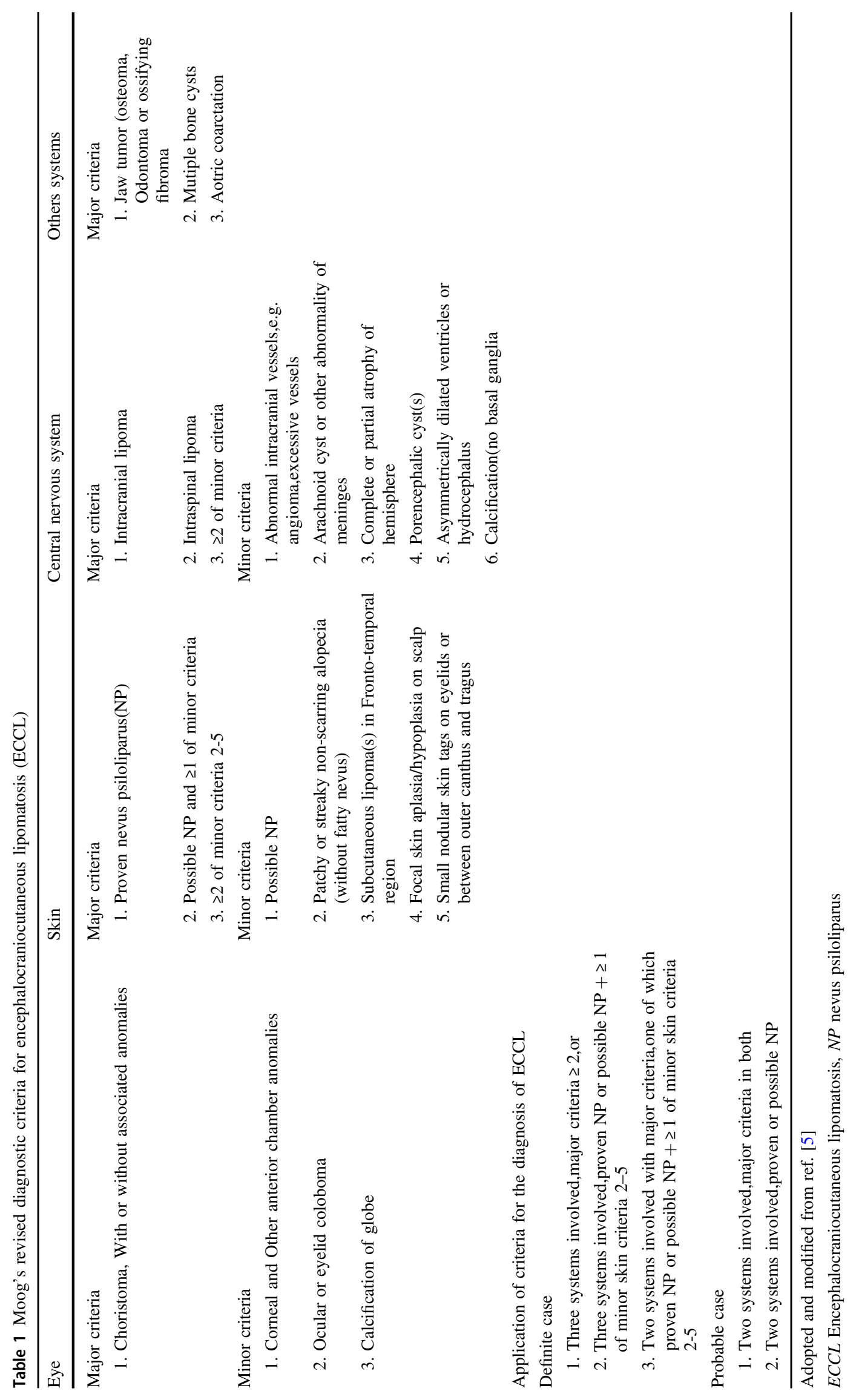


observed in the previous literature. Ophthalmologists should consider the diagnosis of ECCL in their assessment if they encounter patients with these common characteristics.

There is no specific treatment for ECCL. The main ophthalmic approach is surgical removal of local bud tumors, with improvement of the cosmetic appearance as far as possible.

\section{Compliance with ethical standards}

Conflict of interest The authors declare that they have no conflict of interest.

\section{References}

1. Haberland C, Perou M. Encephalocraniocutaneous lipomatosis. A new example of ectomesodermal dysgenesis. Arch Neurol. 1970;22:144-55.

2. Bavle A, Shah R, Gross N, et al. Encephalocraniocutaneous Lipomatosis. J Pediatr Hematol Oncol. 2018. Epub ahead of print.

3. Muñoz-Montufar JP, Rayo-Mares JD, Reyes-Cuayahuitl A. et al. Lipomatosis encefalocraneocutánea: reporte de caso. Gac Med Mex. 2017;153:915-8.

4. Almer Zina, Vishnevskia-Dai Victoria, Zadok David. et al. Encephalocraniocutaneous lipomatosis: case report and review of the literature. Cornea. 2003;22:389-390.

5. Moog U. Encephalocraniocutaneous lipomatosis. J Med Genet. 2009;46:721-9. 